\title{
Turning mobile big data insights into public health responses in times of pandemics: Lessons learnt from the Democratic Republic of the Congo
}

\author{
Chloe Gueguen $^{1, *}$, Nicolas Snel ${ }^{2, *}$ (D) and Eric Mutonji ${ }^{3}$ \\ ${ }^{1}$ The GSMA Foundation AI for Impact (AI4I) Team, Dakar, Senegal \\ ${ }^{2}$ The GSMA Foundation AI for Impact (AI4I) Team, London, United Kingdom \\ ${ }^{3}$ Agence Nationale d'Ingénierie Clinique, de l'Information et de l'Informatique de Santé (ANICiiS), Kinshasa, Democratic \\ Republic of the Congo \\ *Corresponding authors. E-mails: cg.advising@gmail.com; nsnel@gsma.com
}

Received: 30 October 2020; Revised: 07 September 2021; Accepted: 22 October 2021

Key words: Africa; big data; COVID-19; Democratic Republic of the Congo; e-health

\begin{abstract}
Abbreviations: ANICiiS, Agence Nationale d'Ingénierie Clinique, de l'Information et de l'Informatique de Santé; ARPTC, Autorité de Régulation de la Poste et des Télécommunications du Congo; CDR, call detail record; COVID-19, coronavirus disease 2019; DIAL, digital impact alliance; DRC, Democratic Republic of the Congo; FCDO, Foreign, Commonwealth \& Development Office; IOM, International Organization for Migrations; MBD, mobile big data; MNO, mobile network operator; SDG, sustainable development goal; UCD, user-centered design
\end{abstract}

\begin{abstract}
In low-income countries like the Democratic Republic of the Congo (DRC)—where data is scarce and national statistics offices often under-resourced - aggregated and anonymised mobile operators' data can provide vital insights for decision-makers to promptly respond to both prevailing and new pandemics, such as COVID-19. Yet, while research on possible applications of mobile big data (MBD) analytics for COVID-19 is growing, there is still little evidence on how such use cases are actually being adopted by governmental authorities and how MBD insights can effectively be turned into informed public health actions in times of crises. This four-part commentary paper aims to bridge such literature gaps, by sharing lessons learnt from the DRC, whereby Congolese public health authorities, through a steep learning curve, have initiated a public-private sector dialogue with local mobile network operators (MNOs) and their ecosystem partners to leverage population mobility insights for COVID-19 policy-making. After having set the scene on the policy relevance of MBD analytics in the context of the DRC in the first section, the paper will then detail four key enablers that contributed, since March 2020, to accelerate Congolese authorities' uptake of MBD, thus effectively increasing preparedness for future pandemics. Thirdly, we showcase concreate use-cases where "readiness-to-use" has actually translated into actual "usage" and "adoption" for decision-making, while introducing other use cases currently under development. Finally, we explore challenges when harnessing telco big data for decision-making with the ultimate aim to share lessons to replicate the successes and steer the development of MBD for social good in other low-income countries.
\end{abstract}

\section{Policy Significance Statement}

While confirmed cases remain relatively low in Africa, experts warn fragile healthcare systems in many African nations could be overwhelmed in the face of severe COVID-19 outbreaks. Many African countries also face structural flaws in their health information management systems, making it more difficult to navigate through the 
pandemic and allocate appropriate resources and infrastructure needed to fight the COVID-19 pandemic. Through a steep learning curve, the Democratic Republic of Congo (DRC) reached out to mobile network operators to leverage mobility insights to help them manage the health and economic crisis. By sharing key lessons learnt from the DRC, whereby the GSMA, Agence Nationale d'Ingénierie Clinique, de l'Information et de l'Informatique de Santé and their partners have been working toward strengthening the Ministry of Health's readiness-to-use of mobile big data analytics for policy-making, we hope other practitioners across Africa, including governments, donors, and private sector firms, will benefit from these lessons to further increase preparedness for future epidemics.

\section{Setting the Scene}

\subsection{Why leveraging mobile big data analytics in the DRC instead of more traditional data sets?}

In low-income countries like the Democratic Republic of the Congo (DRC) - where reliable data is a scarce resource and national statistics offices are often under-resourced-aggregated and anonymised mobile operators' data can provide vital insights for decision-makers to promptly arbitrate health resources and effectively respond to both prevailing and new epidemics, such as COVID-19. The Democratic Republic of Congo (DRC) is in fact particularly vulnerable to long-standing public health emergencies. The country has been dealing with the world's deadliest measles outbreak, with a total of 382,370 cases and 7,071 deaths between the start of the outbreak on December 31, 2018-September 6, 2020 (WHO, 2020). The Ebola virus disease outbreak in Équateur Province continues to spread, with 40 health areas already affected, while health authorities still struggle to stop a cholera outbreak that has reached 26 health zones (7 provinces), with 13,421 cases and 190 deaths between January 1 and August 9, 2020 (WHO, 2020). On top of this, on March 10, the Minister of Health announced the presence of the first confirmed COVID-19 case in Kinshasa, with a total of 54,863 confirmed cases and 1,059 deaths as of August 31, 2020 (Worldometer, 2020).

In parallel, the DRC continues to experience a complex humanitarian crisis involving armed conflicts and intercommunity tensions resulting in large population movements and increasing numbers of people in need of humanitarian, food and health assistance. At the same time, the country is facing an unprecedented economic slowdown, with the COVID-19 pandemic expected to trigger an economic recession of $-2.2 \%$ in 2020 (IMF, 2020), stemming from weaker exports caused by the global economic downturn, as well as containment measures impacting domestic activities. While the government is actively working with partners to slow the propagation of the COVID-19 epidemics, mitigate its economic impacts, support vulnerable communities ( $72 \%$ of the DRC's population live under the poverty line [WBG, 2020]), and strengthen the resilience of the health system, the overall lack of data remains a major impediment to fight COVID-19 effectively as well as other public health emergencies.

In fact, the DRC has not had a population census since 1984, and following the process of "decoupage" in 2015 that saw the country's existing 11 provinces being split up to 26 (Charnas, 2015), data to inform evidence-based decision-making by province is fairly limited. A consortium led by Grid3 is currently trying to update population estimates for some of the DRC's 26 provinces, while also overlaying these population estimates with the latest health infrastructure data. The importance of this exercise is highlighted by their recent mapping (Grid3, 2020) of villages in the Kongo Central province. While the most comprehensive dataset in the Kongo Central province had only about 500 villages named and located, Grid3's settlement layer featured over 9,000.

Besides, the national health information system still presents numerous weaknesses, with, up-untilrecently, limited harmonization of solutions between directorates and national, provincial and district levels of the Ministry of Health, and multiple paper-based exchanges; therefore, hindering data merging and centralization. Yet, in alignment with the "2016-2020 Plan National de Developement Sanitaire" (National Health Development Plan), a consortium led by the Ministry of Health, with technical support from PATH and Bluesquare, has been working to reinforce the National Health Information System (NHIS), by completing the national DHIS2 system roll-out, creating a data-collection system to report 
health-data to central-levels as well as a platform available in real-time by all layers of the DRC health system.

It is with these data limitations in mind and given the country's ongoing battles with multiple and new diseases, that Congolese public health authorities decided in May 2020 to explore alternative solutions that could help inform data-driven actions and weigh different public health policy trade-offs.

\subsection{How did the collaboration between the mobile industry and health authorities start?}

In May 2020, under the GSMA's initiative and leadership, Agence Nationale d'Ingénierie Clinique, de l'Information et de l'Informatique de Santé (ANICiiS) (the public entity under the Health Ministry in charge of digitizing the health system in the DRC), Kinshasa Digital Academy (a Congolese digital web development and digital communication agency) with the support of Texaf Digital Campus, together with the mobile network operator (MNO) Orange, reached an agreement to collaborate to explore ways mobile big data (MBD) analytics could inform the efforts of the Government of the DRC in responding to COVID-19. The NGO Flowminder Foundation and the MNOs Vodacom Congo and Africell RDC, which were already collaborating to support the presidential COVID-19 Task Force, joined the initiative to support the coordination of the COVID-19 response. All mobile operators agreed to share their aggregated data for free within the scope of this specific data for good initiative and no financial assistance was provided to them. The country's telecommunications regulator, the Autorite de Régulation de la Poste et des Télécommunications du Congo (ARPTC), also endorsed the project, which considerably helped to build trust in the relationship with the mobile operators. Other key partners included: Orange Business Services' Flux Vision, a technical partner providing mobile data analytics based on information from the Orange mobile network; PATH, an NGO advising the Congolese authorities on digital health interventions; Flowminder, an NGO that leverages big data to improve public health and welfare, and the digital impact alliance (DIAL), an organization advancing digital inclusion to achieve the sustainable development goals (SDGs). The work of GSMA and Kinshasa Digital was made possible through the financial support of the Foreign, Commonwealth \& Development Office (FCDO) for GSMA and German Gesellschaft für Internationale Zusammenarbeit (GIZ), Fondation Roi Baudoin and Internews for Kinshasa Digital. The work of Flowminder Foundation has been made possible through the financial support of the Human Security Division, part of the Federal Department of Foreign Affairs of the Swiss Confederation, as well as the Displacement Monitoring Matrix programme of the International Organization for Migration (IOM) in DRC.

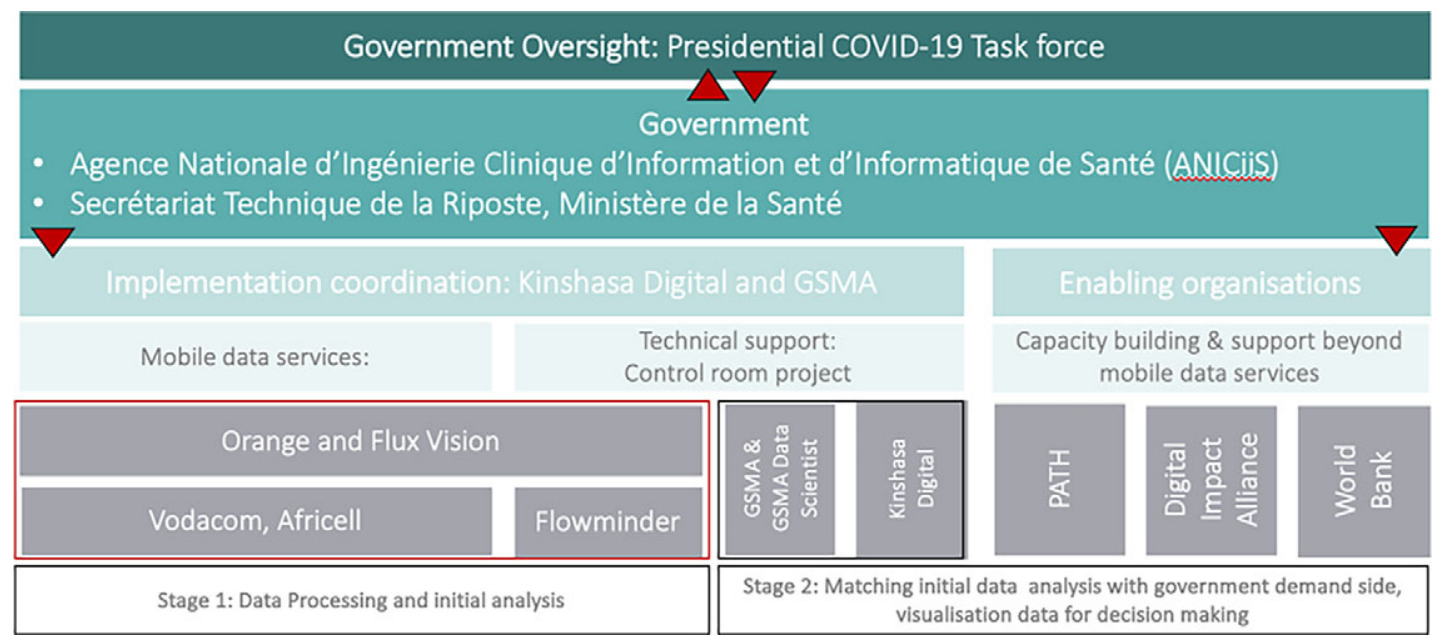

Figure 1. Partners and organization of the ecosystem created in the DRC (nonexhaustive). 
Figure 1 illustrates the ecosystem, in a nonexhaustive way, that has been shaped for this project and the roles and relationships between the main partners involved. GSMA's role has been instrumental to bridging the gap between the government agencies, the mobile operators and the technical partners, coordinating all the parties interested, and moving the project forward. Two weekly meetings were organized, one focused on the technical aspect of the work (Technical Committee) and the other one for general coordination (Control Room), in order to align all partners' objectives and skillsets.

\subsection{How was this public-private sector collaboration framed?}

This collaboration between Congolese health authorities and the mobile industry was framed both via written agreements and regular cross-sector dialogues. In June 2020, ANICiiS addressed an official letter of intent to the GSMA with the dual purpose of, in the immediate term, exploring ways in which MBD could bring value to the COVID-19 response and, in the longer run, seeking ways in which the country could adopt MBD in future decision-making, planning as well as in the evaluation of interventions. In particular, ANICiiS committed to weekly update meetings to discuss the use cases that would drive stronger decision-making, working in close alignment with the GSMA and its partners, and participating in a final workshop to discuss lessons learnt, replicability and sustainability. Such official agreement with the Ministry of Health was complemented with a Membership-agreement that the GSMA already had with all its MNOs. The Flowminder Foundation and Vodacom Congo, partnering in DRC since the 2018 Ebola outbreak, produced a first report presented to the Task Force on May 15. Flowminder and Africell DRC started to collaborate together after this presentation in order to produce coherent mobility metrics across both Vodacom Congo and Africell DRC. GSMA and ANICiiS invited the representatives of the Flowminder Foundation, Vodacom Congo and Africell DRC to join the control room in order to cohesively support the Task Force. Besides, throughout the 5-month collaboration, the GSMA facilitated weekly technical and biweekly coordination calls to align all partners behind common goals, ensure transparency, to support knowledge sharing and to achieve the same level of understanding in the development process of the digital tools. Beyond coordinating the contribution of different mobile operators and their partners, the GSMA also supported this project through sharing lessons learnt from its global AI for Impact initiative, providing data science expertise (including in-country data scientist resources), and ensuring that the project adheres to adequate data privacy standards (GSMA, 2020b).

\section{The Approach: Laying the Foundations for MBD Analytics and Increasing Preparedness for Future Epidemics}

At the start of the COVID-19 pandemic in March 2020, Congolese MNOs had in fact limited previous experience in applying MBD analytics and in leveraging anonymized and aggregated data with third parties. Governmental health authorities also had low awareness and knowledge of MBD. Yet, the Health Ministry, via its newly created e-health agency ANICiiS, quickly understood the potential of MBD to better prepare for future pandemics and saw this partnership with the GSMA and the local digital ecosystem as an opportunity to support the ANICiiS' mandate to apply the power of digital solutions to respond to the unique health challenges the country faces. While MBD analytics are today still in their infancy in the DRC, this project has helped identify a series of success factors, grouped here into four driving forces, that contributed, since March 2020, to accelerating public health authorities' readiness-touse MBD solutions for decision-making (Figure 2).

\subsection{Increasing public health authorities' awareness of what is possible with MBD analytics}

Because MBD concepts were still very new to Congolese authorities back in March 2020, it was important to ensure key stakeholders had a strong foundation of knowledge on what MBD analytics could do and be used for. To do so, the GSMA firstly organized an initial knowledge building workshop in March 2020 with key governmental officials, including the COVID-19 Presidential Taskforce, the Health Ministry's 


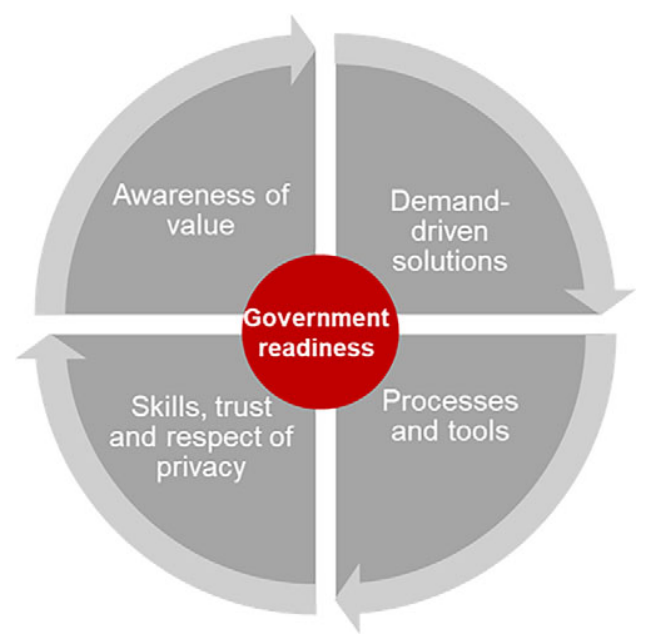

Figure 2. Four driving forces affecting public health authorities MBD readiness-to-use.

COVID-19 Secretariat Technique and ANICiiS, to ensure the same level of understanding of the basics of MBD and key privacy principles, while sharing learnings from previous experiences.

Our agency [ANICiiS] was only effectively launched in February this year, and then, in March, COVID-19 happened. At this time, we did not know anything about Big Data, but in early March, the GSMA, our godfather, approached the government to present the opportunity of using big data to better fight COVID-19. We then created a platform to coordinate with multiple partners: Orange DRC, Africell, Vodacom, Flowminder, and Kinshasa Digital our local digital agency. The GSMA and our other partners really helped us build a strong understanding of where and how mobile big data can bring value.

\section{Ministry of Health. Presentation during the UN World Data Forum, October 2020.}

Besides, setting up weekly "Control Room" and "Technical Committee” meetings was key to aligning multiple stakeholders' interests and recognizing the value of MBD analytics. The first regular virtual meetings, the "Control Room," was the main coordination platform between all the different stakeholders participating in the projects (MNOs, technical providers, governmental agencies, funders ...). The objective of such calls was to align all actors' interests and make sure all major obstacles were promptly identified and collaboratively addressed. Sometimes, these calls were also an occasion for key partners to invite external organizations who, although not directly involved, could potentially open the door for future collaborations (UNICEF, The World Bank, Bluesquare...). The second, the "Technical Committee," as its name suggests, focused on discussing technical development of the solutions. These calls were gathering less participants than the Control Room calls and were happening more frequently, thus allowing key end-users (ANICiiS and the Secretariat Technique) to share their feedback and together decide changes required for the final dashboards. Based on this, data scientists and technical experts from different organizations (GSMA, Kinshasa Digital, Flowminder, and PATH), could exchange knowledge on data processing matters, adapt solutions and agree on future steps for the development of the use cases identified.

\subsection{Developing demand-driven, user-centered MBD solutions}

\subsubsection{Initial assessment to understand key users' needs for insights}

Throughout the project, and while developing technical solutions, the GSMA built upon user-centered design (UCD) techniques to encourage mobile operators and their technical partners to focus on 
putting users - in this case the Ministry of Health via ANICiiS, the Presidential COVID-19 Task Force and the Secretariat Technique - at the center of MBD solutions design and development. At project inception, this started by a need-assessment, led by Kinshasa Digital and the GSMA, which helped identify key insights needs that health authorities expressed to better inform their COVID-19 Response. This included a review of the National COVID-19 Response Plan (Secretariat Technique, Mars, 2020), as well as interviews with the Presidential COVID-19 Task Force, the Secretariat Technique, and ANICiiS. Such assessment exercises highlighted the pressing need for indicators to both better understand population mobility to evaluate the effectiveness of social distancing measures and monitor the hospitals' capacity in Kinshasa.

\subsubsection{The development of $M B D$ analytics solutions}

With a stronger understanding of needs, Kinshasa Digital and Flowminder, together with the GSMA, Orange DRC, Africell, and Vodacom, developed two main MBD solutions that we further detail below.

Mobile data access and processing. Given the serious threat COVID-19 represents for the national health system of the DRC and its prioritization by government, the three mobile operators agreed to participate and contribute to the project by sharing their data without any financial assistance. Orange DRC collaborated directly with their own analytics subsidiary Orange Business Services/Flux Vision to anonymise, process and aggregate the data into mobility Origin-Destination matrices and attendance numbers (number of people present in a zone) at the health district (geographic) level. Some additional mobility data on attendance numbers was also shared at a deeper granular level for preselected hotspots in the capital city of Kinshasa. All the aggregate data was then shared with Kinshasa Digital to build the dashboarding tools. On the other side, Vodacom and Africell worked directly with Flowminder to process and aggregate the data into mobility insights, at the same geographical level as for Orange, for their analytical reports. Data from the different operators has not been combined despite MNOs having different geographical footprints in the country. It is indeed technically challenging to blend data coming from different operators and it does not necessarily add more accuracy since people usually own multiple SIM cards. Nevertheless, models have been compared to each other, when possible, to confirm the trends observed were the same.

Kinshasa Digital's dynamic dashboard on population mobility and hospital capacity, and regular reports. As part of the engagement, Orange DRC in partnership with Orange Business Services/Flux Vision provided aggregated and anonymised data sets to Kinshasa Digital. Then, Kinshasa Digital processed the data to compute the population mobility trends and finally built a nationwide dashboard from scratch which allows health authorities to track population mobility trends within and across different health districts throughout the country. This allows government authorities insight into how movement patterns change in response to government measures, as well as other factors such as economic incentives, or insecurity. As the virus was spreading rapidly from Kinshasa's most affected districts (such as Limete, Gombe, Binza-Meteo, and Binza-Ozone) to other districts in Kinshasa, and to other provinces in the country (most notably Kinshasa, Kongo Central, Sud Kivu, and HautKatanga) amid mining activities resuming, it was a useful tool for the government to track the effect of various interventions (lockdown, closure of schools, etc.) on reducing mobility and to devise informed policy responses. Besides, the dashboard allowed the tracking of population mobility in prelisted "hotpots" (such as universities, religious places, the airport, etc.) in the capital city, provided a highlevel view of hospitals' capacity and, through this, informed required additional communication to the wider public.

Based on the insights from the dashboard, Kinshasa Digital also built regular summary reports highlighting the latest impact on mobility metrics for the authorities. This weekly report aims to quickly deliver an overview of the current situation in terms of mobility change without the need to navigate through the dashboard (Figure 3). 


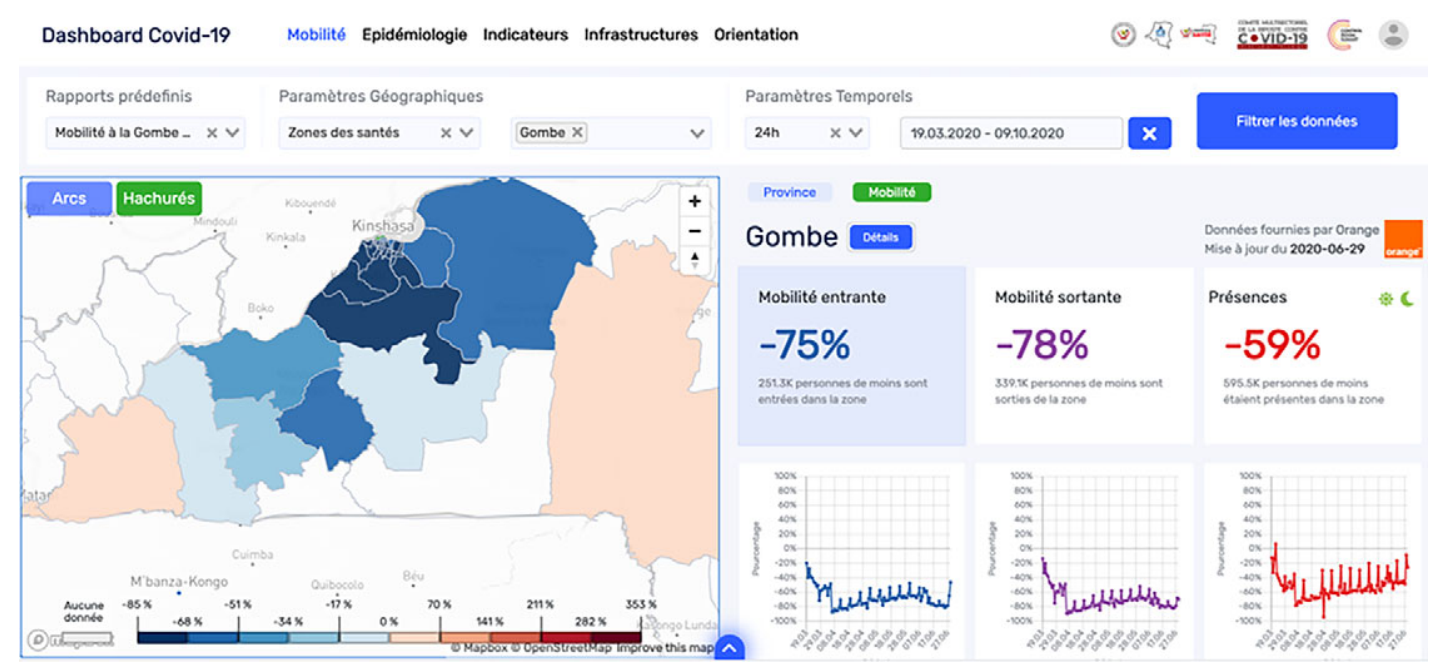

Figure 3. Kinshasa Digital's DRC COVID-19 dashboard. This screenshot taken from the DRC COVID19 dashboard illustrates the insights provided by the tool. In this example, the user visualizes the mobility trends for the Gombe area (neighborhood in Kinshasa) for the period between March 19, 2020 and October 9, 2020. The three metrics on the right-hand side are (from right to left) the attendance (number of people present in the zone during the day or night), the outgoing mobility (number of people leaving Gombe), and the incoming mobility (number of people entering Gombe). The user observes here a sharp decrease for all three indicators in comparison to the period of reference. With the map on the left-hand side, the user here visualizes the incoming mobility trends from all health zones entering Gombe, the blue areas are the ones with a decrease in incoming mobility while the red areas represent an increasing incoming mobility.

Flowminder's reports and open sources codes on population mobility and economic impact measures. In parallel, Vodacom DRC, Africell, and Flowminder have provided government authorities with three reports, which uses aggregated and anonymised call detail records (CDRs) to analyse the impact of the government's confinement measures on mobility patterns in Kinshasa, the capital city. The analysis presented to the Task Force on the May 15, 2020 revealed for instance an overall reduction in population movements between health zones at the national level, and particularly in Kinshasa's Gombe district which saw a drop of $70 \%$ in the total flow of mobile subscribers traveling to Gombe in the weeks after the lockdown, suggesting that adherence to the government's measures was quite high (Figure 4).

The first report (Flowminder, 2020b) also highlights the likely economic impacts of the COVID-19 pandemic in Kinshasa, which saw a drop of the value of mobile phone credit purchases, thus reflecting pressures on household consumption. The report in August notably showed a return toward normal of most mobility indicators, later confirmed in the analysis of October, where a greater focus was put on flows on the entire DRC territory.

Linking MBD analytics to other national data sets. The power of MBD analytics is strengthened when different data sets are combined together producing new insights only visible by joining these different sources of data. This is particularly true for MBD, which on its own, already offers valuable use cases to inform policy makers of the impact of the epidemics, as described above, but can deliver much more if properly merged with additional datasets, such as public health-data or health infrastructure data. In the DRC, we introduced a team of data specialists form the World Bank to the project and all the stakeholders involved, in particular to the mobile operator Orange DRC and the Secrétariat Technique. They gained access to the mobility insights delivered by the dashboards. In combination with other public data sets available they started developing an epidemiological modeling to assess which areas of the country is the 


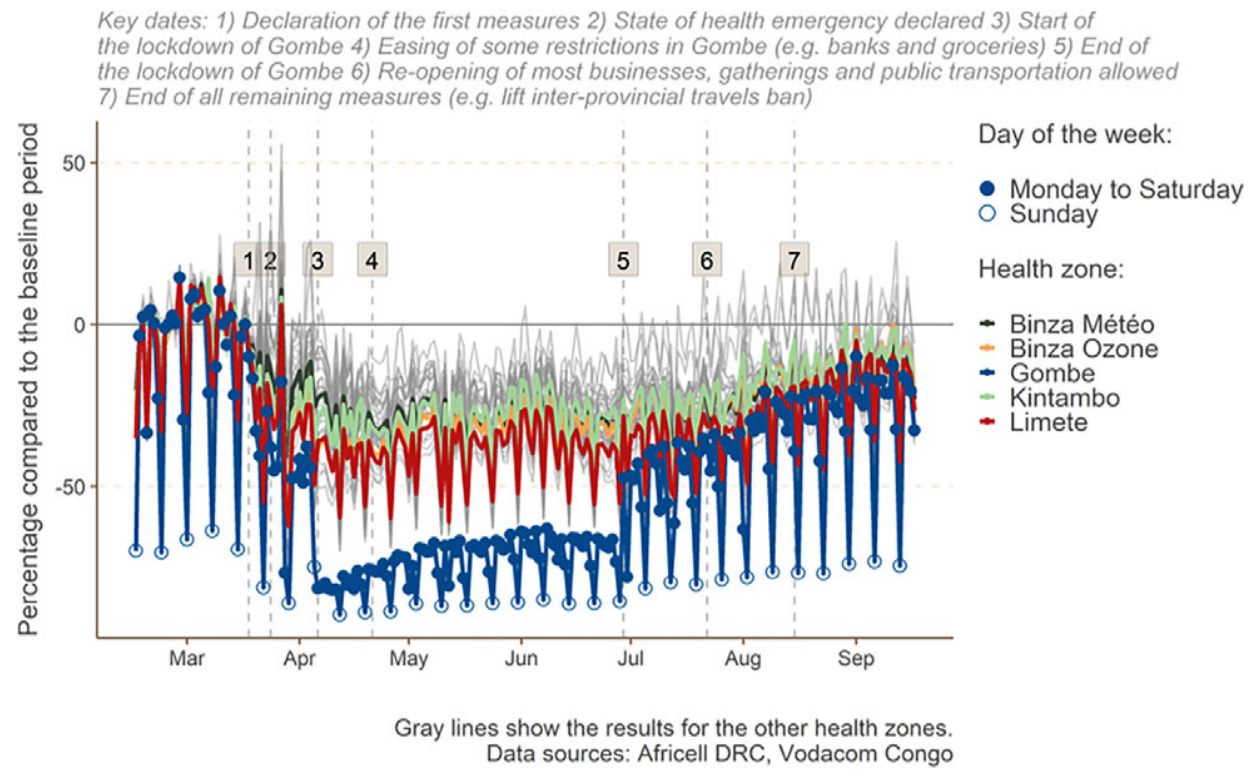

Figure 4. Change (in percentage) of mobility flows from Kinshasa health zones compared to the baseline period.

most at risk. With reference to Figure 5, that described the incremental approach of the use cases deployment in the DRC, every step up comes with a new use case enhancing the value and insights for the end-users thanks to the addition of a new data source. This comes with more complexity in the algorithm developed and eventually in the tools deployed. It is then recommended that the first use cases developed are robust enough in terms of comprehension and methodology before moving up to the next step. It is important to underline here that this incremental approach has been developed with regards to DRC health authorities' priorities and the data availability. In other contexts, it is possible to not follow this incremental approach and, for instance, focus directly on the economic modeling without population mobility data.

\subsection{Creating processes and tools to ensure insights are regularly captured and updated}

The GSMA with the support of Kinshasa Digital implemented a UCD process for the development of the digital tools, through the Technical Committee and Control Room meetings. UCD is a classic product design method based on an iterative process where the developer constantly captures the feedback from the users of the dashboards from a very early stage, listing the different changes to make to the existing solution and prioritizing them.

Developing a web application or interactive dashboard from scratch is extremely time consuming and costly. The only success criteria of such projects are the adoption and the usage rate by the end-users of the dashboards. If the application is barely used, it will become worthless even if the dashboard is beautifully designed and performs seamlessly.

There are two main reasons that can explain a low usage rate of such a digital solution:

- either the app is too complex, and the users lack the skills or the know-how to use it efficiently;

- or the app does not meet the demand of the user.

The reality is often a combination of these two causes.

Usually, the first one, the lack of skills or the nonunderstanding of the app, is the easiest one to fix. For instance, we can offer some extra training to the users to build their digital literacy and make sure they 


\section{Incremental approach to building product and capabilities}

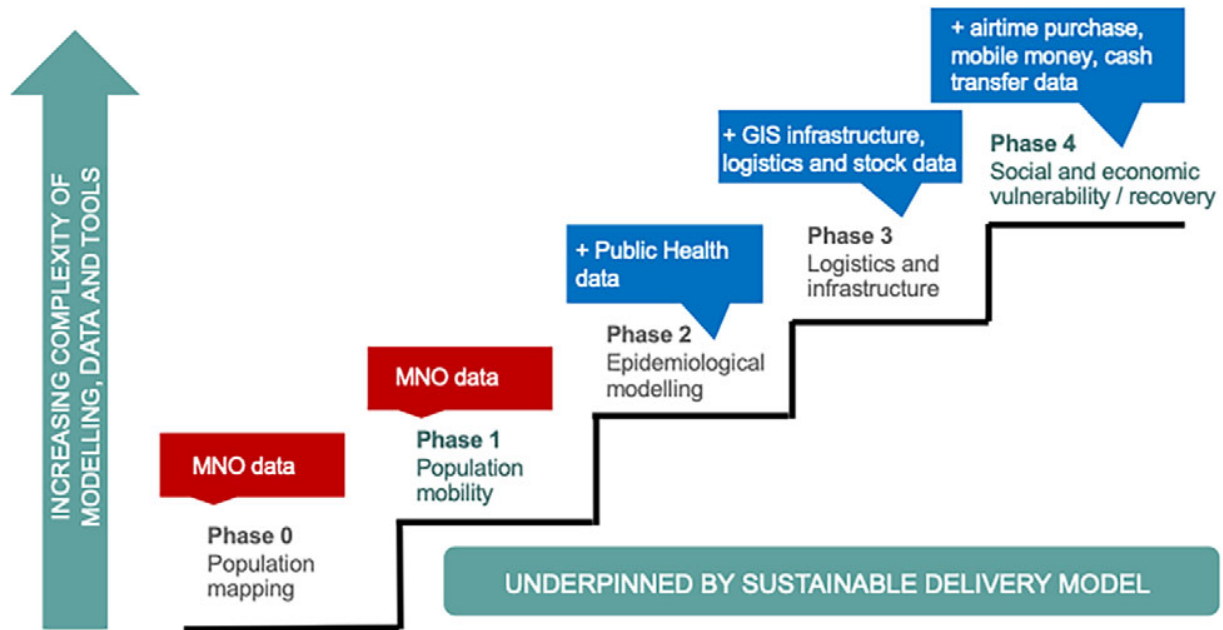

Figure 5. GSMA use case staircase. Phase 0: Population mapping: Before even starting to measure the mobility pattern of the population, it is important to correctly map population, especially in the DRC where the latest census data is from 1984. Mobile phone data can help for this population mapping exercise but usually needs to be combined with other data sources, like satellite imagery. Phase 1:

Population mobility: Mobile phone data can be used to produce aggregate and anonymised population mobility insights to measure the impact of policies on the mobility of the population and also evaluate which areas might be the most at risk given their high mobility patterns. Phase 2: Epidemiological modeling: We add another type of data to the mobility insights: epidemiological data (incidence of the virus, death rate, etc.) and other sociodemographic data available to simulate and better map the risk related to Covid19. How it differs over time and between health districts. Phase 3: WASH infrastructure modeling: Adding another data set related to the country health infrastructure (bed capacity, stock level of medicines, and medical equipment), we can refine the risk model and help prioritize the areas that will need additional WASH stations. Phase 4: Economic modeling: With additional data from mobile recharge and others, it is possible to evaluate the poverty level of the population and the economic impact the

Covid19 has for the people and how it differs between regions.

comprehend how to read and how to play with the dashboard. To that purpose an exhaustive user manual was shared by Kinshasa Digital with all ANICiiS' users to accompany them after the training. Finally, and perhaps most importantly, the application can be redesigned, by adding or removing some features, to make it more accessible or user-friendly to the audience targeted. This is why a regular iterative process is so important, to be able to integrate the changes needed as soon as possible in the process.

The second reason of a low usage rate of a digital application: the nonexisting demand for the existing app, is much harder to tackle. There might be multiple underlying triggers of such a situation and it is important to identify these as quickly as possible in order to find the right solution. For instance, if it is related to mistrust in the data or the algorithm behind it, this can usually be solved by some data validation work or through a technical workshop that explains the data processing and build up confidence of the methodology. However, if the cause of the nonexisting demand appears to be minimal value added to users' work, then some radical actions need to be taken. It could be that the end-users have been wrongly selected and it must then be redirected to the right people or organization that will generate usage of the app. But if it appears the users are the right ones, then the only option left is to pivot the development of the application to fit the demand. This is very different to redesigning the app or adding some extra features, it is really about changing the core of the tool. 
In the DRC, the weekly Technical Committee and Control Room calls allowed us to avoid a pivot situation and anticipated such scenarios by ensuring the application is useful to the user from the very start.

\subsection{Building the MBD ecosystem by strengthening local technical skills and strengthening confidence in the robustness of $M B D$ and trust in the respect of privacy}

One of the major obstacles to adoption of MBD solutions is generally the lack of digital skills of the endusers and often the mistrust in the data and algorithm that comes with it. Testing solutions directly with key governmental users throughout the design process was key not only to help adapt MBD solutions but also to build the government technical skills when using such solutions. Therefore, as part of this project, the GSMA focused on transferring knowledge of MBD to the local workforce, on how to use the application first and also how to build such a solution. These are two distinct skill sets and training usually needs to be carried out for two different groups of people: the future end-users of the application and the next developers/data scientists of MBD solutions.

In October 2020, the GSMA facilitated, together with the ANICiiS and other partners, a series of technical workshops, whereby ANICiiS users got an opportunity to manipulate and share feedback on their user experience of the two solutions developed by Kinshasa Digital and Flowminder. ANICiiS has built upon the technical skills acquired to lead further presentations within the Health Ministry as well as with the Presidential COVID-19 Task Force and other Ministerial departments and Ministries.

Openness and simplicity are key for success. You need to let people express themselves, and you will be surprised how the human brain can approach certain things. I was really impressed and surprised by my own staff members within ANICiiS, on how fast they approach mobile big data analytics.

\section{Ministry of Health. Presentation during the UN World Data Forum, October 2020}

\section{Outcomes and Impact}

\subsection{Early usage of population mobility tools by governmental agency and development of new use cases}

Although MBD services are still in their infancy in the DRC, we are observing initial signs of MBD adoption among public health authorities. In this section, we showcase concreate examples where "readiness-to-use" has actually translated into actual "usage" and "adoption" for decision-making, particularly when it comes to evaluating the adherence of lockdown measures across the country, identifying hotspots and monitoring health infrastructure capacity in the capital city of Kinshasa.

The dashboard shows that the strategic decision to lock down the country and the city of Gombe was an important decision, although one that was made without scientific evidence based on data. The dashboard now gives confidence to the members of the Presidential Task Force to make informed decisions in the future.

\section{Presidential Covid-19 Task Force, DRC}

This is an important tool for us and I thank in advance all partners and the donors who will help us to keep improving the dashboard so that we can use it even more now with this second wave.

\section{Secrétariat Technique du Covid-19, DRC}

Following the adoption and the first usage phase of the mobility dashboard by the health authorities, we have identified, through our regular feedback sessions, a series of additional use-cases where further 
technical work will be needed. Such use cases include: (a) an epidemiological model to track and predict the spread of the disease under various scenarios so that the healthcare system could be better prepared; (b) a WASH dashboard where mobile data can be leveraged to optimize where Hygiene, Sanitation and Water stations are positioned and where Health Information may need to be intensified; (c) an alert system, whereby designated "community-relays agents," who are local health agency representatives, could be informed in case of abnormal situations thus ensuring COVID-19 responses are promptly cascaded from national to regional health levels; and (d) mobility insights relative to travelers returning to DRC, especially given the current context of reopening of borders.

Most of the future use cases identified need integration with other data sources and require specific data science and modeling expertise to process the data properly, for which we are actively seeking collaboration with academics and other organizations.

\subsection{Empowerment of ANICiiS, as the leading agency to build a National Health Information System and digitally transform the health panorama in DRC}

Through various trainings, workshops and handover sessions, the ANICiiS team has increasingly gained skills and confidence in terms of data literacy and data strategy. The agency is now able to manipulate the dashboards, to train other people how to use them and eventually to promote such digital tools to other governmental agencies and to an external audience. In fact, the young e-health governmental agency is now actively promoting MBD tools not only within the Ministry of Health (COVID-19 and EBOLA Secretariat Technique) but also with the Presidential COVID-19 Taskforce, other working groups (such as the UNICEF CASS working group) and other Ministries (such as the Ministry of Scientific Research). Although not always directed to the "primary/direct" users of the COVID-19 MBD tools, such dissemination events with external audiences help increase awareness of the value of digital solutions to inform decision-making and will help replicability of such solutions in the context of other diseases and challenges within health areas and beyond. It is indeed possible to leverage MBD and adapt the dashboard already developed for other outbreaks, where human mobility is an important factor, such as Ebola for instance.

We can now use mobile big data to inform the decisions of tomorrow. Mobility indicators can assist our government to make decisions, not only with COVID-19, because we also have in DRC other longstanding diseases such as Ebola and Malaria which have been here for decades... Tomorrow, mobile big data will help our Ministry to make informed decisions, allocate resources and better fight these pandemics.

Ministry of Health. Presentation during the UN World Data Forum, October 2020

Besides, continuous knowledge transfer between ANICiiS and the Control Room digital experts throughout this MBD project, has also indirectly supported ANICiiS in fast-tracking the development of other e-health solutions to quickly respond to COVID-19's pressing challenges. This included the launch of a national "Stop Coronavirus COVID-19 DRC" official public website and a smartphone application "STOP Coronavirus," which provides the population transparency on COVID-19 numbers, clarity on key governmental measures, hygiene and health advice, while demystifying some fake ideas related to COVID-19; an increased contact tracing tools and enhanced mapping of field resources via GIS data, thanks to the collaboration of ANICiiS with GRID3 and PATH; and deployment of chatbots to relay automatic responses to some of the most regular questions raised by Congolese citizens, in real-time and based on their geographic location.

Ultimately, the fast-tracked digital developments described above and achieved since the start of the pandemic, have also indirectly pushed ANICiiS to strengthen its governance structures. In fact, ANICiiS has now developed clear internal processes to validate the development of future digital tools, thus ensuring developments are aligned with the e-Health National Plan (ANICiiS, 2020) and associated 
budgets. In November 2020, ANICiiS reviewed the terms of references of all existing e-health technical solutions in DRC, and MBD Analytics have been highlighted as priority solutions that will be presented during upcoming donor roundtables.

\subsection{Initial discussions to build an adequate legal framework to ensure the protection of personal data and confidentiality principles}

While all these MBD developments are part of ANICiiS's efforts to build a National Health Information System and further advance toward Universal Health Coverage, the use of big data generally raises questions when it comes to ensuring data privacy. In general, if not overseen properly, the use of personal data from various sources could pose privacy, confidentiality and ethical risks. To reinforce the importance of individual privacy as a fundamental right and to avoid any forms of discrimination, all Control Room partners under this MBD project agreed to the GSMA COVID-19 Privacy Guidelines (GSMA, 2020b) at the start of the project. To further embed privacy controls, ANICiiS is currently drafting a Data Privacy Charter that future governmental technical partners will need to abide by. In parallel, ANICiiS is initiating discussions, together with nation legal bodies, to work toward the creation of a legal framework and national laws to protect data privacy and trust in the future.

\section{Key Lessons Learned}

This experience in the DRC showed that harnessing telco big data for decision-making also can come with challenges. In DRC, such challenges included a complex ecosystem, limited internet connectivity, limited national statistics and relatively nascent digital data bases, limited in-country technical resources, and dedicated budgets, for governmental big data and artificial intelligence projects, etc.

Although the project is still under development, with new use-cases being added to the tools already created and ANICiiS further promoting adoption within the Ministry of Health and other Ministries, this initiative allowed the identification of eight initial learnings that we have regrouped under the four driving forces, identified in Section 2.2, accelerating the government ability to use MBD solutions. We hope these learnings will be able to inform future MBD developments.

The awareness of value

- Make it concrete, demonstrate "value" and "impact": at the start of the project, it was difficult for stakeholders to "project" MBD work too far ahead. Decision-makers needed to see some results before moving forward but mockups, proof of concepts and technical workshops helped a lot to generate feedback and discussions. This advocacy work is necessary with the mobile operators as well, at the highest level if possible, which often lack the full understanding of the potential of their data.

- Engage early and widely with all national and international stakeholders to improve coordination from day 1 . Our access to the presidential taskforce has been essential for our credibility and to accelerate the adoption process of the tools developed. Including the telecom regulator in the discussions from the early stages will also significantly help in the long run with the relationships with the mobile operators.

Skills, trust, and respect of privacy

- Empower key users and co-create locally: Understanding local complexities between various health agencies and identifying the key decision-makers is essential, especially in contexts like the DRC with complex political contexts. Working with local stakeholders is also key to increasing the chance of sustainability. In this project frame, this was achieved by ensuring the GSMA and other international organizations were not trying to influence but rather co-create and co-shape digital changes together with local stakeholders such as ANICiiS and Kinshasa Digital. 
- Mobile data is highly sensitive information and needs to be treated carefully from the early stages to avoid any relationship damage between the government, their citizens and the MNOs. Any projects involving a data sharing agreement must adhere to adequate data privacy standards (GSMA, 2020b).

\section{Processes and tools}

- Collaborate and promote openness: It is important to build a collaborative and open platform whereby everyone can exchange ideas and learnings. Such open platforms can also help identify synergies between multiple datasets.

- Keep things simple: Adopting an incremental approach and building use-cases in a gradual manner, is key to ensuring buy-in and adoption from stakeholders. It was key to share knowledge on each step of the journey, including by supporting skills and talent development, defining processes and tools, thus maximizing government readiness and the chance of adoption.

\section{Demand-driven solutions}

- Empathize: It is important to spend sufficient time to "empathize" and understand different stakeholders to build a fruitful ecosystem collaboration. Interviewing every stakeholder individually to understand their concerns, priorities and needs at the start of, and throughout the collaboration is key. This is also true for the mobile operators, spending time to understand what they can gain in return for their collaboration, such as building big data expertise in house for instance or strengthening relationships with their regulator.

- Keep an eye on your watch, focus on sustainability and replicability: Balancing evolving health priorities (COVID-19 vs. other deadlier diseases) with longer technical MBD solution developments was a challenge. To bridge such issues, it was key to ensure continuous dialogue between the technical team and the solution users, while engaging (not too late) on discussions on the replicability and sustainability of such tools for other diseases.

\section{Conclusion}

While MBD services are still in their infancy in the DRC, this paper aimed to share lessons on ways to accelerate the uptake of MBD so that such services can effectively be quickly adopted to sharpen leadership and decision-making, both in times of crisis and in normal times. Steering MBD for policymaking requires a multifaceted approach which needs to be adapted to local contexts and viewed as an ongoing journey. By sharing key success factors and lessons learnt from DRC, we aim to provide guidance on how public-private partnerships can initiate this journey to support the development of a sustainable MBD for social good level playing field in other low-income countries.

Acknowledgments. The authors are grateful for the project support provided by all stakeholders from the Control Room, namely: ANICiiS, the GSMA, Orange, Africell, Vodacom, Kinshasa Digital, Flowminder, PATH, and DIAL.

Funding Statement. This research was supported by a grant from the Foreign, Commonwealth \& Development Office (FCDO), under the GSMA AI4I Project. The funder had no role in study design, data-collection and analysis, decision to publish, or preparation of the manuscript.

Competing Interests. The authors declare no competing interests exist.

Author Contributions. Conceptualization: C.G., N.S.; Methodology: C.G., N.S.; Supervision: E.M.; Writing-original draft: C.G., N.S.; Writing - review and editing: N.S.

Data Availability Statement. The mobility data that support the findings of this study were made available from mobile operators Orange DRC and Orange Business Services to Kinshasa Digital and GSMA, and from mobile operators Vodacom and Africell to Flowminder Foundation. Restrictions apply to the availability of these data, which were used under license for this study. Data sharing requests can be made and will be examined by mobile operators themselves. All codes developed are openly accessible on Github. The dashboards developed by Kinshasa Digital are only accessible with credentials, that can be requested to them (Flowminder, 2020a; GSMA, 2020a; Kinshasa Digital, 2020; Kinshasa Digital Academy ,2020).

Supplementary Materials. To view supplementary material for this article, please visit http://doi.org/10.1017/dap.2021.30. 


\section{References}

ANICiiS (2020) Plan National de Développement de l'Informatique de Santé 2020-2024. Available at https://www.researchgate. net/publication/344042425_Plan_National_de_Developpement_de_l'Informatique_de_Sante_en_RDC_2020-2024 (accessed March 2021).

Charnas F (2015) Decoupage in the DRC: The art of redecorating political landscapes. Available at https://www.dailymaverick. co.za/article/2015-07-06-decoupage-in-the-drc-the-art-of-redecorating-political-landscapes/ (accessed March 2021).

Flowminder (2020a) Codes to Process Mobility Aggregate from Mobile Operators is Open Source. Available at https://github.com/ Flowminder/COVID-19/tree/c9b81d2af6404af2a5c78f0b71bcee9dcc867279 (accessed March 2021).

Flowminder (2020b) Democratic Republic of the Congo (DRC): Initial Insights into the Effect of Mobility Restrictions using Anonymised and Aggregated Mobile Phone Data. Available at https://www.flowminder.org/media/mghdallt/flowminder_initi alreportcovid-19_vodacomdrc_eng.pdf (accessed March 2021).

Grid3 (2020) New dataset released for one of the most populous provinces in the Democratic Republic of the Congo. Available at https://grid3.org/news/new-dataset-released-for-kongo-central (accessed March 2021).

GSMA (2020a) Codes to Process Mobility Aggregate Data Received from Orange is Open Source. Available at https://github.com/ djeffkanda/GSMA (accessed March 2021).

GSMA (2020b) The GSMA COVID-19 Privacy Guidelines, April. Available at https://www.gsma.com/publicpolicy/wp-content/ uploads/2020/04/The-GSMA-COVID-19-Privacy-Guidelines.pdf (accessed March 2021).

IMF (2020) IMF Country Report No 20/146. Available at https://www.imf.org/-/media/Files/Publications/CR/2020/English/ 1CODEA2020001.ashx (accessed March 2021).

Kinshasa Digital (2020) Codes to Visualise Mobility Impact from Mobility Aggregate Data Received from Orange and Processed by GSMA is Open Source. Available at https://github.com/DRC-COVID19/stopcoronavirus.cd (accessed March 2021).

Kinshasa Digital Academy (2020) Stop Coronavirus Rdc Dashboard. Available at https://www.stopcoronavirusrdc.info/dash board-maps/landing (accessed March 2021).

Secretariat Technique COVID-19 (2020) Plan de Preparation et de Riposte contre l'Epidemie au COVID-19 en Republique Democratique du Congo.

WBG (2020) Country Overview, Democratic Republic of Congo. Available at https://www.worldbank.org/en/country/drc/over view (accessed March 2021).

WHO (2020) Weekly Bulletin on Outbreaks and Other Emergencies. Available at https://apps.who.int/iris/bitstream/handle/10665/ 334353/OEW38-1420092020.pdf (accessed March 2021).

Worldometer (2020). Available at https://www.worldometers.info/coronavirus/country/democratic-republic-of-the-congo/ (accessed March 2021).

Cite this article: Gueguen C, Snel N and Mutonji E (2022). Turning mobile big data insights into public health responses in times of pandemics: Lessons learnt from the Democratic Republic of the Congo. Data \& Policy, 4: e8. doi:10.1017/dap.2021.30 